\title{
Biogenesis and biological implications of isomiRs in mammals- a review
}

\author{
Jasdeep Kaur Dhanoa, Ramneek Verma, R. S. Sethi, Jaspreet Singh Arora and C. S. Mukhopadhyay * (D)
}

\begin{abstract}
Background: IsomiRs, the sequence-variants of microRNA (miRNA), are characterized by variation at the 3'- and/or $5^{\prime}$-end(s) of canonical miRNA-sequence as a result of nucleotide addition or deletion or substitution. These sequence alterations could be created either due to imprecise cleavage of miRNA sequence by drosha or dicer enzymes or through the addition of nucleotides at $3^{\prime}$ end during miRNA-biogenesis.

Main body: The present review elaborates the biogenesis vis-à-vis role of isomiRs in disease-related traits in human and animals. The differential expression of isomiRs has been detected in the early and late developmental phases during embryogenesis in fruit fly and halibut (Hippoglossus hippoglossus). Multidimentional role of isomiRs viz. in gene regulation, evolution, RNA interference pathway and differentiation of tumorous cells etc. has attracted researchers to explore the biological significance of isomiRs in different species. Biocomputational identification of isomiRs using suitable online software/tools (miR-isomiRExp, miRPro, isomiRBank, isomiR-SEA etc) has been followed by empirical validation and pathway analyses.
\end{abstract}

Conclusion: IsomiRs have been associated with various disease-pathways and thus could be used as promising disease-related markers in humans and livestock. In addition, the involvement of isomiRs in cancer and other diseases has been the major topic of interest due to the involvement of different biogenesis pathways.

Keywords: IsomiR, Disease-marker, Livestock, miRNA, Biological pathways

\section{Background}

MicroRNAs constitute a family of small non-coding RNA molecules (of 20-25 nucleotides length) that do not code for protein. Most of them are present in the introns while some exist in the exons of a gene. They play a major role in gene expression regulation and RNA silencing. The dysregulation of any miRNA may be associated with diseases like cancer or heart disease. Similarly, the isomiRs that are defined as the variants of miRNA sequence could have evolved through various cellular processes. The sequence variation in the isomiRs arises through different processes including addition or deletion etc. in the canonical miRNA sequence. In this review, we will discuss isomiRs, their biogenesis, functional involvement in various diseases and some databases that can be used for their prediction.

\footnotetext{
* Correspondence: csmbioinfo@gmail.com

School of Animal Biotechnology, Guru Angad Dev Veterinary and Animal Sciences University, Ludhiana, Punjab 141004, India
}

\section{MicroRNA}

MicroRNAs (miRNAs) are the post-transcriptional regulators of gene expression in most of the eukaryotic cells. These miniature RNAs belong to a family of small ( 2022 nucleotides in length) non-coding RNA-molecules that are expressed in wide range of organisms including plants, animals, and worms [1]. The miRNA-mediated RNAinterference was first discovered in 1993 by Ambros and coworkers while working on lin-4 gene involved in larval development of Caenorhabditis elegans (C. elegans) [2, 3]. The expression of about $30 \%$ of the protein-coding genes [4-7] is regulated by these non-coding RNA sequences through binding to the 3 '-untranslated regions (3'-UTR) of specific mRNAs.

Over a thousand of miRNA-encoding genes are present in the human genome [8], which are directly or indirectly associated with more than $30 \%$ of the protein coding genes $[9,10]$. The miRNAs precursors are not found within the coding regions of transcripts or the corresponding antisense strand, instead, they are present in clusters within intergenic regions and introns of protein-coding 
genes with some exceptions such as hsa-miR-935, hsamiR-302 [11]. The biosynthesis of miRNAs differs in plants and animals. In plants, the entire process of biogenesis of primary-miRNA (pri-miRNA) to mature miRNA occurs in the nucleus by RNase III enzyme DCL1 (Dicer-like 1). While in animals, the process occurs in the cytoplasm as well as in nucleus by two different enzymes: Drosha/Pasha in nucleus and Dicer in the cytoplasm $[3,12]$. A vast array of miRNA-repertoire has been identified and reported in different animal and plant species. Recently, in our laboratory, we have identified novel bubaline-miRNAs (using small RNA sequencing and analysis) [13-15] and also by biocomputational analysis of whole genome shotgun sequences [16]. It has also been reported that target genes of differentially expressed miRNAs (from healthy as well as diseased individuals) can have a critical role in innate immunity and TLR (Toll like receptors) signaling pathways [14]. An array of isomiRs is the isoforms of a miRNA indicating that these might be functionally important. The present review aims at elaborating the biogenesis and features of isomiRs, and their roles in biological processes, with special emphasis on diseases in animals.

\section{IsomiRs}

A single miRNA could differ by a small number of base changes (due to insertion/deletion or substitution) at the $5^{\prime}$ or $3^{\prime}$ termini, resulting in the formation of isomers of specific miRNA, termed as $5^{\prime}$ or $3^{\prime}$ isomiRs, respectively [17]. Thus, isomiRs are derived by imprecise cleavage or any change in processing of mature miRNA from primary transcripts by Drosha and Dicer enzymes and nucleotide addition at 3' end [18]. IsomiRs can also be generated through RNA editing and single nucleotide polymorphisms (SNPs) from the canonical (any molecular sequence that represents the consensus sequence of that particular molecule) miRNA sequence $[18,19]$.

Prior to proceeding further on isomiRs, it is necessary to discuss in brief about the nomenclature of miRNAs and isomiRs and their features. There are different ways of writing the names of miRNAs based on the nomenclature. For example- "MIR" refers to the gene that encodes miRNA, "miR" refers to the mature form of miRNA and "mir" refers to the pre-miRNA and the pri-miRNA [20]. Similarly, there are various terms used for isomiRs on basis of their appearance and for analysis purpose. Table 1 adumbrates some of these terminologies that pertain to isomiRs (Table source: https://bioinfo2.ugr.es/miRanalyzer/miRanalyzer_tutorial.html).

\section{Biogenesis of isomiRs}

IsomiRs are generated by post-transcriptional modifications of the corresponding miRNA sequence. isomiRs have diverse roles in animals, plants, and viruses.
Table 1 Terminologies related to isomiRs

\begin{tabular}{|c|c|c|}
\hline S.No & IsomiR name & Description* \\
\hline 1 & $5^{\prime}$ isomiR & Base change (deletion or addition) at $5^{\prime}$ terminus \\
\hline 2 & $3^{\prime}$ isomiR & Base change (deletion or addition) at $3^{\prime}$ terminus \\
\hline 3 & $\mathrm{RC}$ & $\begin{array}{l}\text { Read count (used for NGS read counts after } \\
\text { sequencing transcriptome or sRNA) }\end{array}$ \\
\hline 4 & UR & $\begin{array}{l}\text { Unique reads of a particular miRNA or isomiR or } \\
\text { transcript }\end{array}$ \\
\hline 5 & 3SNE & Single nucleotide extension in $3^{\prime}$ end \\
\hline 6 & $3 \mathrm{MNE}$ & Multiple nucleotide extension in $3^{\prime}$ end \\
\hline 7 & 3Trim & $\begin{array}{l}\text { the read is shorter than the reference mature } \\
\text { sequence but it starts at the same position in } \\
\text { the hairpin }\end{array}$ \\
\hline 8 & $5 \mathrm{MNE}$ & Multiple nucleotide extension in $5^{\prime}$ end \\
\hline 9 & 5Trim & $\begin{array}{l}\text { the read aligns to a position in the hairpin } \\
\text { after the reference mature sequence BUT } \\
\text { the last base of the read and the mature } \\
\text { sequence map to the same position }\end{array}$ \\
\hline 10 & Mature star & Less expressed arm of the pre-miRNA \\
\hline 11 & Predom UR & $\begin{array}{l}\text { the number of unique reads of the } \\
\text { predominant mature microRNA sequence }\end{array}$ \\
\hline 12 & Predom RC & $\begin{array}{l}\text { the read count of the predominant mature } \\
\text { microRNA sequence }\end{array}$ \\
\hline 13 & Star RC & $\begin{array}{l}\text { the read count of the predominant mature } \\
\text { microRNA sequence }\end{array}$ \\
\hline 14 & Star UR & $\begin{array}{l}\text { the number of unique reads of the } \\
\text { predominant mature microRNA sequence }\end{array}$ \\
\hline 15 & IsoRC & IsomiR read count \\
\hline 16 & Exact RC & $\begin{array}{l}\text { the read count of perfectly mapped } \\
\text { miRBase consensus sequences }\end{array}$ \\
\hline 17 & 3SNA & Single nucleotide addition in $3^{\prime}$ \\
\hline 18 & 3 MNA & Multiple nucleotide addition in $3^{\prime}$ \\
\hline 19 & RPM & Reads per Millions \\
\hline 20 & Other LV & $\begin{array}{l}\text { The number of other length variants } \\
\text { (those that have length variation in } 5^{\prime} \text { and } 3^{\prime} \text { ) }\end{array}$ \\
\hline
\end{tabular}

*Table source: https://bioinfo2.ugr.es/miRanalyzer/miRanalyzer_tutorial.html

To understand the biology of isomiRs, it's required to adumbrates the process of miRNA production in animals. miRNA biogenesis occurs through different steps in the cytoplasm and nucleus. The process starts in the nucleus where precursor miRNA (pre-miRNA) is transcribed from miRNA coding gene by RNA polII/III, which is then cleaved into shorter sequences by Drosha/DGCR8 complex to form primary-miRNA. The pre-miRNA is then exported from nucleus to cytoplasm by Exportin-5. Mature miRNA is formed from this precursor miRNA through miRNA/miRNA duplex formation. This precursor miRNA is also involved in the formation of isomiRs [21, 22]. Alternatively different pathways can also be involved that can alter the sequenceof canonical miRNA. Further, the isomiRs can be $5^{\prime}$-isomiRs or $3^{\prime}$-isomiRs (Fig. 1). It is evident that the 'miRNA: miRNA duplex' can form 


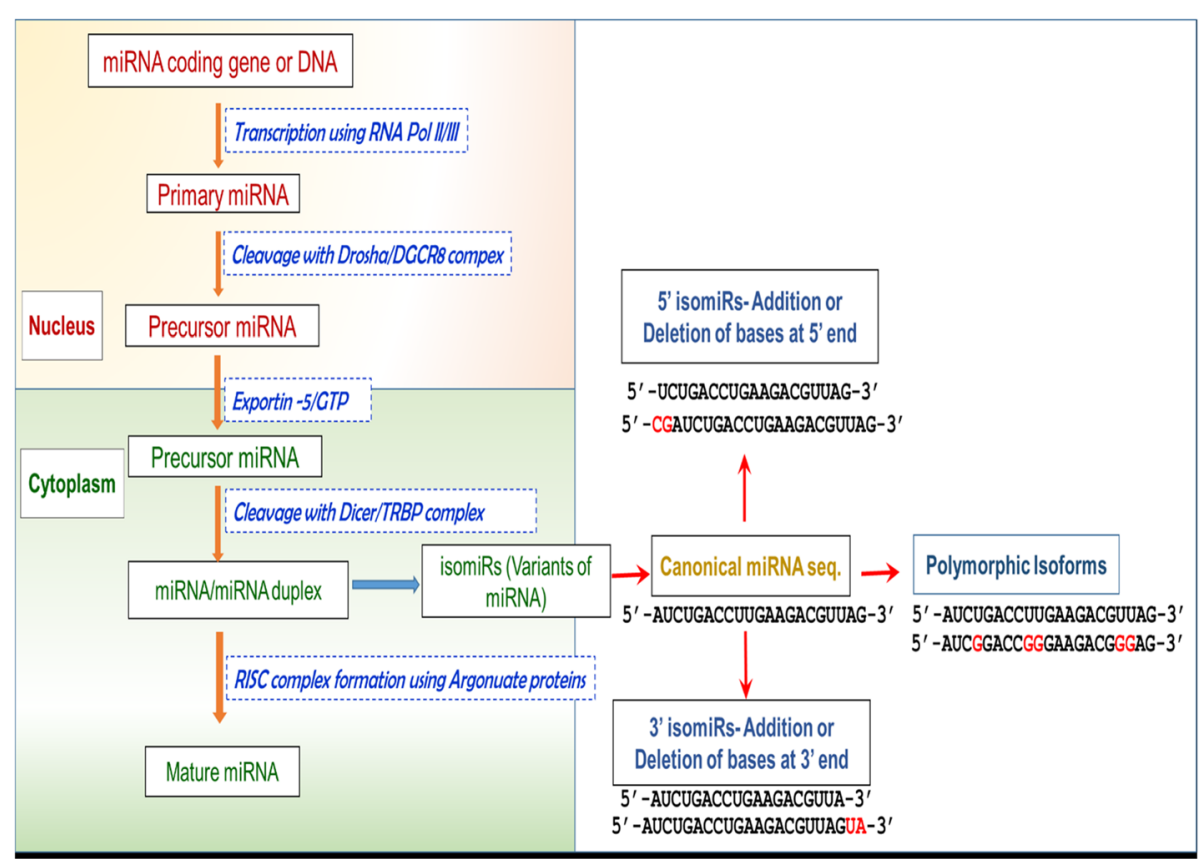

Fig. 1 Flow-diagram to demonstrate the biogenesis of miRNA and its variants (isomiRs) in normal adult cells of an animal (The sequence used here is arbitrary for explanation purpose only)

through complementary nucleotide base pairing of miR-5p and miR-3p generated from pre-miRNA [19].

Based on variation in the canonical sequence, isomiRs can be template dependent or non-template dependent. In the template dependent, $5^{\prime}$ or $3^{\prime}$ nucleotide shifting occurs due to exoribonuclease, which ultimately results in the formation of isomiRs. On the other hand, in non-template dependent variation results in post-transcriptional RNA editing and tailing. Adenosine to Inosine (A to I) is commonly occurring RNA editing and diverse set of nucleotidyltransferases are responsible for post-transcriptional tailing. Both these events carry out the formation of isomiRs [23] (Fig. 2).

It has been consistently observed that the $5^{\prime}$-ends of the miRNAs are uniform that results in the formation of 5 '-isomiRs due to different characterized 5 '-ends. Thus, in comparison to miRNA with a single seed, the miRNA with $5^{\prime}$-isomiRs could have a significantly diverse target range and functional influence. These types of isomiRs have been recognized in many species including fruit fly (Drosophila melanogaster), mice (Mus musculus) and human [24]. The significance of $5^{\prime}$-isomiR expression on miRNA target repertoires was studied on vertebrate miR-142-3p by Manzano and coworkers [25]. They reported that 5 '-isomiRs that differ from each other by 1 nucleotide can have highly divergent or convergent target ranges. Similarly, any variation in the $3^{\prime}$ end of miRNA can alter the stability and the efficiency of target repression of miRNA [26] (Fig. 2).

\section{Evolutionary perspective of isomiRs}

The evolutionary pattern of different miRNA families in divergent species can provide information on functional adaptations and associations in various species of plants and animals. Some of the researchers have provided information on the evolutionary perspective of isomiRs in different taxa. A recent study conducted in vivo on mammalian cells has shown that, Dicer can alternatively process these miRNAs to generate $5^{\prime}$-isomiRs. This alternative Dicer processing can be the result of the sliding bulge in the pre-miRNAs indicating that secondary structure of miRNAs can be responsible for the generation of 5'-isomiRs [27]. Tan and team [28] demonstrated through Northern blot analysis that a proportion of individual miRNA in human may vary spatially. Besides, the team also analyzed the isomiRs from miRBase and inferred that during evolution, canonical miRNAs have been replaced by $5^{\prime}$ isomiRs. This finding supports the hypothesis that isomiRs are the procreation of evolutionary relices of the miRNA genes. The expression profiles of miRNA and isomiRs can be regulated through arm selection (miRNAs may be derived from different arms) or arm switching (two arms usually exhibits dynamic expression patterns). It has been reported that two arms miR-3p and miR-5p contribute to different evolutionary/expression patterns of miRNA and isomiRs [29]. The structural regions within the miRNA (mature miRNA or seed loop) can be under evolutionary pressure. However, the miRNAs that are present within 


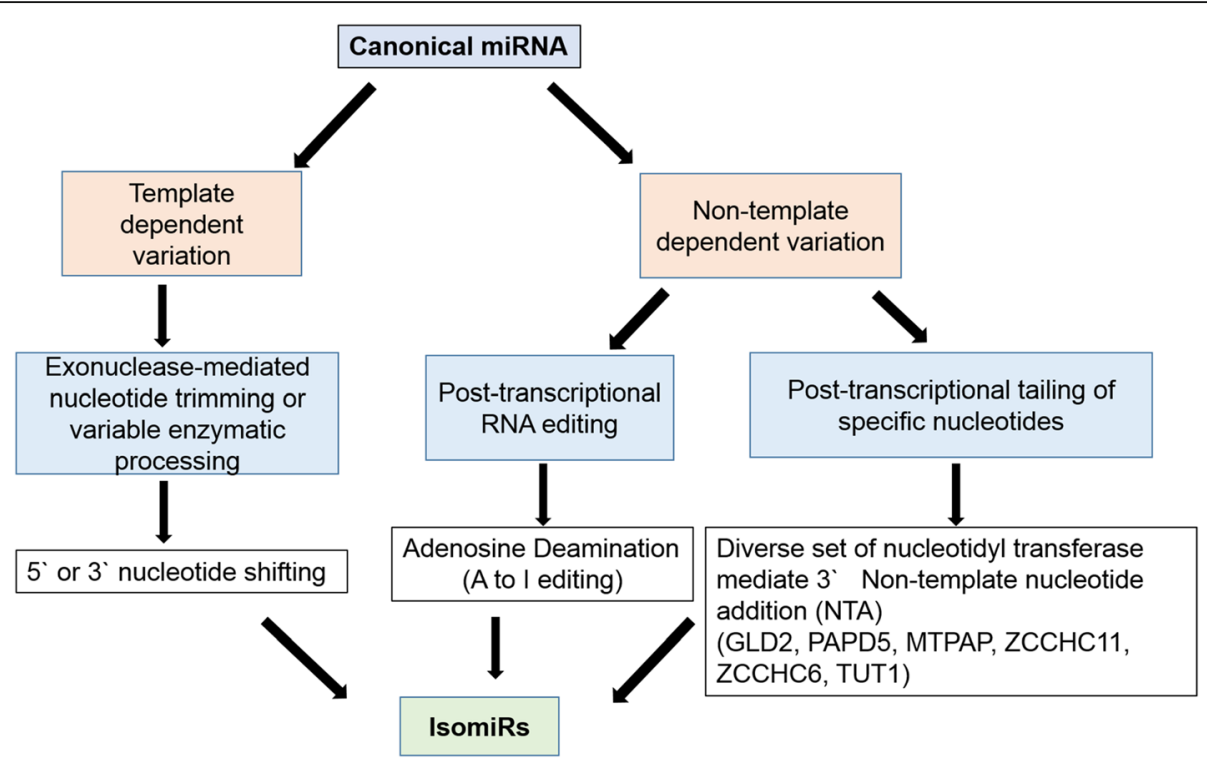

Fig. 2 Different methods of generation of isomiRs from canonical miRNA. During miRNA biogenesis, the canonical miRNA can have template dependent and non-template dependent variations which result in synthesis of miRNA variants known as isomiRs

miRNA clusters (miRNA in close proximity of other miRNA) can share similar structures including seed sequences. Gene ontology and analysis of miRNA clusters can provide insight information regarding molecular functions of these clusters. However, evolutionary conservation of isomiRs warrants further in depth study and exploration [30].

\section{Importance of isomiRs}

IsomiRs act as potential regulatory molecules and are associated with repression of the target-mRNAs [31]. Several isomiRs are directly involved in post-transcriptional gene silencing, and could also affect the homologous miRNA itself. For example, the isomiRs may increase stability of miRNA and modify the effectiveness of miRNAs through RISC (RNA-induced silencing complex) during maturation process [32]. The small RNA (sRNA) deep-sequencing data showed that isomiRs are differentially expressed in developmental genes and tissues of fruit fly (Drosophila melanogaster). The addition of 'A' or ' $U$ ' at 3 ' end of miRNA during early or late embryogenesis suggests that the stability of miRNA or miRNA: target interactions get strengthened. [33].

It has been reported that conserved target sites for isomiRs can be predicted by using biocomputational analysis. A study using immunoprecipitation showed that isomiRs can be incorporated into argonaute proteins and from the luciferase assay it has been observed that isomiRs are different from their canonical miRNA on a functional basis [34]. To study the features and regulatory targets of mature miRNAs, a comprehensive analysis was performed on
Arabidopsis. The computational analysis revealed that isomiRs play a key role in gene regulation via terminal heterogeneity, which ultimately enhances the specificity of target gene silencing. The analysis of degradome data available in this experiment indicates the biological role of isomiRs in target cleavage. Further, the comparison of novel predicted and validated target genes with commonly targeted mRNA genes from Gene Ontology (GO) demonstrated that the validated targets are bound to isomiRs along with canonical miRNA [35]. IsomiRs have also been identified in human lymphoblastoid cell lines whose expression was population specific as well as gender-dependent. It has been reported that these isomiRs participate in RNA interference (RNAi) pathway through their association with Argonaute silencing complex [36].

Chan and colleagues [37] reported that not all, but some isomiRs may share common mRNA targets. They investigated isoforms of miR-31 $(\mathrm{miR}-31 \mathrm{H}, \mathrm{miR}-31 \mathrm{P}$ and miR-31 M) that differ slightly at 5' and 3' ends. The study investigated concordant and discordant regulation, displayed by 6 known target genes (CEBP $\alpha$, E2F2, STK40 etc) of isomiR-31. The results revealed that in cellbased systems, isomiRs exhibit similar and disparate regulation of target genes. Moderate level of isomiR expression has been observed between Dicer-independent miRNA and non-dominant miRNA, suggesting complex miRNA maturation process at isomiR level [38]. The study performed by Mercey and colleagues [39] on human miR-34/449 family suggested that isomiR variants which differ by single canonical counterpart can 
share biological functions indicatind additional mechanism by which regulation of complex biological function can be perfectly and easily employed by miRNA machinery.

\section{Role of isomiRs in human diseases}

The relationship of isomiR expression and disease progression is not clear till date. However, some reports are there which show some association of isomiRs with different diseases. In the case of Alzheimer's disease (AD), a significant change in miRNA isoforms was found between early and late stages of the disease. An entropy based MIH5 model was introduced to identify effects of dysregulation of miRNA isoforms at $5^{\prime}$ end. The results indicated that as compared to the expression-based method, the entropy-based method is most stable to detect miRNA related to AD [40]. Using miR-183-5p in breast cancer and normal breast data sets of 2 races (white and black) from the Cancer Genome Atlas repository (https://www.cell.com/pb-assets/consortium/pancanceratlas/pancani3/index.html), it has been reported that as compared to archetype miRNA, full isomiR profile from known and novel human-specific miRNA can provide better results to distinguish between normal and tumorous tissueas its isomiRs were upregulated in breast cancer related white women but not black. Also, a distinct impact of these isomiRs on cellular transcriptome has been identified due to overexpression of isomiRs in MDAMB- 231 cells followed by microarray analysis [41].

The overexpression of canonical miRNA hsa-miR-140-3p and its 5 'isomiR-140-3p has been reported in breast cancer patients. Reduced cell viability was observed in breast cancer cell lines (MCF10A, MDA-MB-468, and MDAMB-231) due to higher expression of 5 'isomiR-140-3p. The cell cycle analysis showed that 5 'isomiR-140-3p caused decreased cell migration and cell cycle arrest at G0/G1 phase. The data revealed that 5'isomiR-140-3p contributes to tumor-suppressive effects by reducing breast cancer proliferation and migration, [42]. In another study, small RNA (sRNA) sequencing databases were used to study the expression of miRNA and isomiRs by correlating with gender difference. It was determined that the expression of miRNA can vary between different tissues and genders. From the statistical analysis they concluded that there is a significant difference between the expression of miRNA and isomiRs in tumor and normal tissues in both sexes. The study suggested that screening of miRNA/isomiRs associated with the disease could be affected by gender difference [43].

Babapoor and team [44] identified isomiR sequence of miRNAs that were deregulated in cutaneous melanomaand found that miR-451a was functioning as a tumor suppressor in gastrointestinal cancer cells and glioma cells. They concluded that miR-451a was involved in melanoma progression while the mature form of this isomiR, miR-451a.1 was associated with amelanotic melanoma [44]. Zhang and coworkers [45] developed a new method to discover the catalogue of isomiRs in association with cancer progression in human. Differentially expressed isomiRs were detected using DEseq algorithm, followed by rank based MANOVA. The expression pattern between normal and tumorous tissues when compared using MANOVA algorithm and the biological functions of isomiRs when elucidated using functional enrichment analysis. There was significant inconsistency in the expression of multiple isomiRs derived from same miRNA locus in normal and tumor samples .

The miRNA transcriptional response of human dendritic cells to various mycobacterium infections showed that bacterial infection has a strong impact on the cellular immune response of host which can alter the expression and proportion of miRNA isoforms. A total of 1595 isomiRs corresponding to 235 miRNAs were detected with alteration in the expression due to bacterial infection using DESeq algorithm (which is based on negative binomial distribution). isomiRs. It has been observed that due to bacterial infection, as compared to start site, the end regions of isomiRs show great variability. The miRNA repertoire involved in providing immunity to Mycobacterium tuberculosis was also identified using deep sequencing [46, 47]. The results of transcriptome and miRNA analyses of human peripheral blood mononuclear cells (PBMCs) were also used to identify the isomiR profile. The results from data entered in miRBase indicated that the most abundant isomiR sequences did not match the reference miRNA sequence. This specifies that there is a dynamic change in the relative expression level of isomiRs derived from the same precursor (which may vary depending on cell type and its differentiation status) [48].

\section{IsomiRs in livestock}

A very limited research has been conducted on role of isomiRs in livestock. The study on tooth morphogenesis in miniature pigs (Sus scrofa) revealed that out of 11 unique miRNA sequences, a total of five (mir-103, mir-107, mir-133a, mir-133b, mir-127) belong to isomiR families and play an important role in developmental stages of teeth including incisors, canines, bicuspids and molars [49]. The miRNA study during bovine oestrous cycle revealed the presence of isomiRs in bovine plasma using sRNAbench. A total of 655 isomiRs were identified from 134 canonical miRNA with modifications on 3 '-end, $5^{\prime}$-end and also in the middle of canonical miRNA. Some of the isomiRs were showing higher expression level than their canonical sequence suggesting that some bovine miRNA do not correspond to their isoforms [50]. In case of cattle some isomiRs were showing $3^{\prime}$ or $5^{\prime}$ end 
variation, but some isomiRs, for example, miR-125-p showed both $3^{\prime}$ as well as $5^{\prime}$ end variations. IsomiRs have also been found in porcine muscle cell study (miR-423) and in longissimus muscle of sheep (miR-96) at 3 ' end variants [51]. The results from Illumina deep sequencing of pig miRNA and isomiRs sequence revealed its role during early pregnancy [52]. The miR$127-3 p$ is one of the highly expressed miRNA in retina and RPE/Choroid. The deep sequencing study conducted on mouse retina indicated the presence of similar level of miR-127-3p and its isomiRs [53]. In a recent study novel miRNAs and their orthologs has been detected in left ventricular wall of rat heart [54]. Transcriptome sequencing analysis performed on murine HL-1 cells identified that $5^{\prime}$ isomiRs target the genes that are involved in cardiovascular disease. In the same study 2 identical genomic loci of miR-133a $5^{\prime}$ isomiR (i.e. miR-133a-1 and miR-133a-2) were identified in mammals that process different loci [55].

\section{IsomiR databases}

IsomiR Bank (http://mcg.ustc.edu.cn/bsc/isomir/) is a free online database created to integrate detected isomiRs. According to the data provided by Zhang and coworkers [56], a total of 308,919 isomiRs collected from 4706 mature miRNA are present in isomiR Bank. This bank provides the analysis of target prediction and enrichment for evaluating isomiRs effects on target selection. Another online platform miR-isomiRExp has been developed recently to analyze the expression of specific miRNA at miRNA/ isomiR level. This software can reveal functional characteristics and can provide the whole mechanism involved in maturation and processing of miRNA/isomiR. This platform also provides information regarding deregulated miRNA loci and detailed isomiR sequence [57].

There are various tools available to predict and quantify known and novel miRNA including miRDB (http://mirdb.org/), miRFinder (http://www.bioinformatics.org/mirfinder/), and miRDeep2 (https://www.mdc-berlin.de/content/mir deep2-documentation). Another software miRPro (https:// sourceforge.net/projects/mirpro/ > Browse) has been developed recently which is able to predict novel miRNA and can quantify the known miRNA. This software can also detect isomiRs, which is not possible with miRDeep2. It also includes genome annotation based read count, optional seed region check, expression quantification of miRNA and arm switching detection [58]. It has been reported that many tools do not provide information regarding isomiRs of specific miRNA and conserved miRNA-mRNA interaction sites. To overcome these problems Urgese and colleagues [59] introduced a novel software named isomiR-SEA. This software characterizes the seed presence of miRNA in input tags and evaluates the position of mismatches. Thus it recognizes isomiRs and characterizes the interaction sites for miRNA-mRNA.

\section{Conclusion}

Isoforms of miRNA may provide detailed information about the specific cell or tissue type. The miRNAs and their variants can be used in disease association and detection in different species by studying differential expression pattern. However, more detailed and in-depth studies are warranted to explore the usability of isomiRs as markers for important diseases in animals and humans.

\section{Abbreviations \\ AD: Alzheimer's disease; C. elegans: Caenorhabditis elegans; DCL1: Dicer-like 1; GO: Gene ontology; miRNA: MicroRNA; NGS: Next generation sequencing; PBMC: Peripheral blood mononuclear cells; RISC: RNA induced silencing complex; SNP: Single nucleotide polymorphism; sRNA: Small RNA; TLR: Toll like receptors; UTR: Untranslated region}

\section{Acknowledgments}

The a-uthors thankfully acknowledge Dr. Simrat Sagar Singh, Dean, Postgraduate Studies, Guru Angad Dev Veterinary and Animal Sciences University Ludhiana for providing facilities.

\section{Funding}

The authors are thankful to the Department of Biotechnology (DBT), Government of India (Project no. AAB-009) for providing funds.

Availability of data and materials

Not applicable.

\section{Authors' contributions}

JKD: Manuscript data collection and writing; CSM: data collection and manuscript drafting; JSA, Ramneek and RSS: drafting and revising the manuscript critically. All authors read and approved the final manuscript.

Ethics approval and consent to participate

Not applicable.

Consent for publication

Not applicable.

Competing interests

The authors declare that they have no competing interests.

\section{Publisher's Note}

Springer Nature remains neutral with regard to jurisdictional claims in published maps and institutional affiliations.

Received: 19 April 2017 Accepted: 27 December 2018

Published online: 14 February 2019

\section{References}

1. Ramesh SP. MicroRNA function: multiple mechanisms for a tiny RNA. RNA. 2005; 11:1753-61.

2. Lee RC, Feinbaum RL, Ambros $V$, Ambros F. The $C$. elegans heterochronic gene lin-4 encodes small RNAs with antisense complementarity to lin-14. Cell. 1993;75(5):843-54. https://doi.org/10.1016/0092-8674(93)90529-Y.

3. Wahid F, Shehzad A, Khan T, Kim YY. MicroRNAs: synthesis, mechanism, function, and recent clinical trials. Biochim Biophys Acta. 2010;1803:1231-43.

4. Enright AJ, John B, Gaul U, Tuschl T, Sander C, Marks DS. MicroRNA targets in Drosophila. Genome Biol. 2003;5:R1. https://doi.org/10.1186/ gb-2003-5-1-r1.

5. Lewis BP, Burge CB, Bartel DP. Conserved seed pairing, often flanked by adenosines, indicates that thousands of human genes are microRNA targets. Cell. 2005;120:15-20. 
6. Alexandra D, lliopoulos D. MicroRNA gene networks in oncogenesis. Curr Genomics. 2009;10(1):35-41. https://doi.org/10.2174/138920209787581299.

7. Friedman RC, Farh KK, Burge CB, Bartel DP. Most mammalian mRNAs are conserved targets of microRNAs. Genome Res. 2009:19:92-105.

8. Liu W, Mao S-Y, Zhu W-Y. Impact of tiny miRNAs on cancers. World J Gastroenterol. 2007;13(4):497-502

9. John B, Enright AJ, Aravin A, Tuschl T, Sander C, Marks DS. Human microRNA targets. PLoS Biol. 2004;2:e363. https://doi.org/10.1371/journal.pbio.0020363.

10. Grun D, Wang YL, Langenberger D, Gunsalus KC, Rajewsky N. microRNA target predictions across seven Drosophila species and comparison to mammalian targets. PLoS Comput Biol. 2005;1:e13. https://doi.org/10. 1371/journal.pcbi.0010013.

11. MacFarlane LA, Murphy PR. MicroRNA: biogenesis, function and role in Cancer. Curr Genomics. 2010;11:537-61.

12. Mukhopadhyay CS, Singh J. MicroRNAs: basics, implications and modulation in improving disease resistance, DBT sponsored short term training course on molecular tools and bioinformatics approaches for livestock genome analysis, delivered on 13th September 2014 at central Institute for Research on cattle, grass farm road, Meerut, UP, organized during 10th to 30th September; 2014. p. 67-73.

13. Singh J, Mukhopadhyay CS, Kaur S, Malhotra P, Sethi RS, Choudhary RK. Identification of the MicroRNA repertoire in TLR ligand challenged bubaline PBMCs as a model of bacterial and viral infection. PLoS One. 2016;11(6): e0156598. https://doi.org/10.1371/journal.pone.0156598.

14. Dhanoa JK, Singh J, Singh A, Arora, JS, Sethi RS, Mukhopadhyay CS. Discovery of isomiRs in PBMCs of diseased vis-à-vis healthy Indian water buffaloes. Accepted in ExRNA.

15. Mukhopadhyay CS, Verma R, Singh J. Extraction and $\mathrm{qPCR}$ based detection of miRNAs from cultured PBMCs of bubaline origin. In: Mossman K, editor. Innate antiviral immunity: methods and protocols: Publisher Humana Press; 2017. p. 1656. https://doi.org/10.1007/978-1-4939-7237-1. eBook ISBN: 978-14939-7237-1, Hardcover ISBN: 978-1-4939-7236-4, Series ISSN: 1064-3745.

16. Manku HK, Dhanoa JK, Kaur S, Arora JS, Mukhopadhyay CS. Biocomputational identification of microRNAs from bubaline whole genome shotgun sequences. Comput Biol Chem. 2017;70:96-106. https://doi.org/10.1016/j.compbiolchem. 2017.08.005.

17. Neilsen CT, Goodall GJ, Bracken CP. IsomiRs-the overlooked repertoire in the dynamic microRNAome. Trends Genet. 2012;28:544-9.

18. Morin RD, O'Connor MD, Griffith M, Kuchenbauer F, Delaney A, Prabhu AL, Zhao Y, McDonald H, Zeng T, Hirst M, et al. Application of massively parallel sequencing to microRNA profiling and discovery in human embryonic stem cells. Genome Res. 2008;18:610-21.

19. Guo L, Zhang H, Zhao Y, Yang S, Chen F. Selected isomiR expression profiles via arm switching. Gene. 2014;533(1):149-55.

20. Wright MW, Bruford EA. Naming junk: human non-protein coding RNA (ncRNA) gene nomenclature. Hum Genomics. 2011;5(2):90-8. https://doi. org/10.1186/1479-7364-5-2-90.

21. Lages E, Ipas H, Guttin A, Nesr H, Berger F, Issartel J-P. MicroRNAs: molecular features and role in cancer. Front Biosci. 2012;17:2508-40.

22. Zou Q. Presentation on theme: computational prediction of miRNA and miRNA-disease relationship. China: Professor School of Computer Sci\&Tech Tianjin University; 2015.

23. Vickers KC, Sethupathy P, Baran-Gale J, Remaley AT. Complexity of microRNA function and the role of isomiRs in lipid homeostasis. J Lipid Res. 2013; 54:1182-91.

24. Ruby JG, Stark A, Johnston WK, Kellis M, Bartel DP, Lai EC. Evolution, biogenesis, expression, and target predictions of a substantially expanded set of Drosophila microRNAs. Genome Res. 2007;17:1850-64.

25. Manzano M, Forte E, Raja AN, Schipma MJ, Gottwein E. Divergent target recognition by co-expressed 5'-isomiRsof miR-142-3p and selective viral mimicry. RNA. 2015;21(9):1606-20.

26. Wyman SK, Knouf EC, Parkin RK, Fritz BR, Lin DW, Dennis LM, et al. Posttranscriptional generation of miRNA variants by multiple nucleotidyl transferases contributes to miRNA transcriptome complexity. Genome Res. 2011;21:1450-61.

27. Ma H, Wub Y, Niu Q, Zhang J, Jia G, Manjunath N, Wua H. A sliding-bulge structure at the dicer processing site of pre-miRNAs regulates alternative dicer processing to generate5'-isomiRs. Elsevier. 2016;2(9):e00148. https:// doi.org/10.1016/j.heliyon.2016.e00148.

28. Tan GC, Chan E, Molnar A, Sarkar R, Alexieva D, Isa IM, Robinson S, et al. 5' isomiR variation is of functional and evolutionary importance. Nucleic Acids Res. 2014;42(14):9424-35. https://doi.org/10.1093/nar/gku656.
29. Guo L, Yu J, Yu H, Zhao Y, Chen S, Xu C, Chen F. Evolutionary and expression analysis of miR-\#-5p and miR-\#-3p at the miRNAs/isomiRs levels. Biomed Res Int. 2014a;2015:1-14.

30. Chen L, Heikkinen L, Knott KE, Liang Y, Wong G. Evolutionary conservation and function of the human embryonic stem cell specific miR-302/367 cluster. Comp Biochem Physiol. 2015;16:83-98.

31. Cloonan N, Wani S, Xu Q, Gu J, Lea K, Heater S, Barbacioru C, et al. MicroRNAs and their isomiRs function cooperatively to target common biological pathways. Genome Biol. 2011;12:R126.

32. Ebhardt HA, Tsang HH, Dai DC, Liu YF, Bostan B, Fahlman RP. Meta-analysis of small RNA-sequencing errors reveals ubiquitous post-transcriptional RNA modifications. Nucleic Acids Res. 2009;37:2461-70.

33. Fernandez-Valverde SL, Taft RJ, Mattick JS. Dynamic isomiR regulation in Drosophila development. RNA. 2010;16:1881-8.

34. Tan GC, Nicholas D. IsomiRs have functional importance. Malaysian J Pathol. 2015;37(2):73-81.

35. Ahmed F, Senthil-Kumar M, Lee S, Dai X, Mysore KS, Zhao PX. Comprehensive analysis of small RNA-seq data reveals that combination of miRNA with its isomiRs increase the accuracy of target prediction in Arabidopsis thaliana. RNA Biol. 2014;11(11):1414-29.

36. Loher P, Londin ER, Rigoutsos I. IsomiR expression profiles in human lymphoblastoid cell lines exhibit population and gender dependencies. Oncotarget. 2014;5(18):8790-802.

37. Chan Y-T, Lin Y-C, Lin R-J, Kuo H-H, Thang W-C, Chiu K-P, Yu AL. Concordant and discordant regulation of target genes by miR-31 and its isoforms. PLoS One. 2013;8(3):e58169.

38. Liang T, Yu J, Liu C, Guo L. IsomiR expression patterns in canonical and dicer-independent microRNAs. Mol Med Rep. 2017;15(3):1071-8.

39. Mercey O, Popa A, Cavard A, Paquet A, Chevalier B, Pons N, Magnone V, et al. Characterizing isomiR variants within the microRNA-34/449 family. FEBS Lett. 2017;591(5):693-705.

40. Wang $S$, Xub Y, Li M, Tub J, Lub Z. Dysregulation of miRNA isoform level at $5^{\prime}$ end in Alzheimer's disease. Gene. 2016;584:167-72.

41. Telonis AG, Loher P, Jing Y, Londin E, Rigoutsos I. Beyond the one-locusone-miRNA paradigm: microRNA isoforms enable deeper insights into breast cancer heterogeneity. Nucleic Acids Res. 2015;43(19):9158-75.

42. Salem O, Erdem N, Jung J, Münstermann E, Wörner A, Wilhelm H, Wiemann S, Körner C. The highly expressed 5'isomiR of hsa-miR-140-3p contributes to the tumor suppressive effects of miR-140 by reducing breast cancer proliferation and migration. BMC Genomics. 2016;17:566. https://doi.org/ 10.1186/s12864-016-2869-x.

43. Guo L, Liang T, Yu J, Zou Q. A comprehensive analysis of miRNA/isomiR expression with gender difference. PLoS One. 2016;11(5):e0154955.

44. Babapoor S, Fleming E, Wu R, Dadrasm SS. A novel miR-451a isomiR, associated with Amelanotypic phenotype, acts as a tumor suppressor in melanoma by retarding cell migration and invasion. PLoS One. 2014;9(9):e107502.

45. Zhang H, Yang S, Guo L, Zhao Y, Shao F, Chen F. Comparisons of isomiR patterns and classification performance using the rank-based MANOVA and 10-fold cross-validation. Gene. 2015:569:21-6.

46. Siddle KJ, Tailleux L, Deschamps M, Loh YHE, Deluen C, Gicquel B, et al. Bacterial infection drives the expression dynamics of microRNAs and their isomiRs. PLoS Genet. 2015;11(3):e1005064.

47. Mukhopadhyay CS, Verma R, Singh J. Extraction and gPCR based detection of miRNAs from cultured PBMCs of bubaline origin. In: Innate antiviral immunity: Series- Methods Mol Biol. [Ed. Mossman K.] Springer Publication; Humana Press, New York, USA. 2017. 1656:89-102. https://doi.org/10.1007/978-1-4939-7237-1_4.

48. Vaz C, Ahmad HM, Bharti R, Pandey P, Kumar L, Kulshreshtha R, Bhattacharya A. Analysis of the microRNA transcriptome and expression of different isomiRs in human peripheral blood mononuclear cells. BMC Research Notes. 2013;6:390.

49. Li A, Li Y, Song T, Wang F, Dayong Liu D, Fan Z, Cheng S, Zhang C, Wang J, He J, Wang S. Identification of differential microRNA expression during tooth morphogenesis in the heterodont dentition of miniature pigs, Sus Scrofa. BMC Dev Biol. 2015;15:51.

50. Ioannidis J, Donadeu FX. Circulating microRNA profiles during the bovine Oestrous cycle. PLoS One. 2016;11(6):e0158160. https://doi.org/10.1371/ journal.pone.0158160.

51. Fatima A, Morris DG. MicroRNAs in domestic livestock. Physiol Genomics. 2013;45:685-96.

52. Krawczynski K, Bauersachs S, Reliszko ZP, Graf A, Kaczmarek MM. Expression of microRNAs and isomiRs in the porcine endometrium: implications for 
gene regulation at the maternal-conceptus interface. BMC Genomics. 2015; 16:906. https://doi.org/10.1186/s12864-015-2172-2.

53. Soundara Pandi SP, Chen M, Guduric-Fuchs J, Xu H, Simpson DA. Extremely complex populations of small RNAs in the mouse retina and RPE/choroid. Invest Ophthalmol Vis Sci. 2013;54:8140-51. https://doi.org/10.1167/iovs.13-12631.

54. McGahon MK, Yarham JM, Daly A, Guduric-Fuchs J, Ferguson LJ, Simpson DA, Collins A. Distinctive profile of IsomiR expression and novel MicroRNAs in rat heart left ventricle. PLoS One. 2013;8(6):e65809. https://doi.org/10. 1371/journal.pone.0065809.

55. Chiang HR, Schoenfeld LW, Ruby JG, Auyeung VC, Spies N, et al. Mammalian microRNAs: experimental evaluation of novel and previously annotated genes. Genes Dev. 2010;24:992-1009.

56. Zhang Y, Zang Q, Xu B, Zheng W, Ban R, Zhang H, Yang Y, Hao Q, lqbal F, Li A, Shi Q. IsomiR Bank: a research resource for tracking IsomiRs. Bioinformatics, 2016;32(13):2069-71.

57. Guo L, Yu J, Liang T, Zou Q. miR-isomiRExp: a web-server for the analysis of expression of miRNA at the miRNA/isomiR levels. Sci Rep. 2016a;6:23700.

58. Shi J, Dong M, Li L, Liu L, Luz-Madrigal A, Tsonis PA, Rio-Tsonis KD, Liang C. mirPRo-a novel standalone program for differential expression and variation analysis of miRNAs. Sci Rep. 2015;5:14617.

59. Urgese G, Paciello G, Acquaviva A, Ficarra E. isomiR-SEA: an RNA-Seq analysis tool for miRNAs/isomiRs expression level profiling and miRNA-mRNA interaction sites evaluation. BMC Bioinformatics. 2016;17:148.

Ready to submit your research? Choose BMC and benefit from:

- fast, convenient online submission

- thorough peer review by experienced researchers in your field

- rapid publication on acceptance

- support for research data, including large and complex data types

- gold Open Access which fosters wider collaboration and increased citations

- maximum visibility for your research: over $100 \mathrm{M}$ website views per year

At $\mathrm{BMC}$, research is always in progress.

Learn more biomedcentral.com/submissions 